Pacific Journal of Mathematics

A SPECIAL DEFORMATION OF THE METRIC WITH NO
NEGATIVE SECTIONAL CURVATURE OF A RIEMANNIA
SPACE

Grigorios TSAGAS 


\title{
A SPECIAL DEFORMATION OF THE METRIC WITH NO NEGATIVE SECTIONAL CURVATURE OF A RIEMANNIAN SPACE
}

\author{
Grigorios Tsagas
}

The main results of this paper can be stated as follows. Let $M_{1}, M_{2}$ bc two big open submanifolds of the Riemannian manifolds $\left(\boldsymbol{R}_{1}^{2}, h_{1}\right)$ and $\left(\boldsymbol{R}_{2}^{2}, h_{2}\right)$, respectively. The submanifolds $M_{1}, M_{2}$ with the metrics $h_{1} / M_{1}$ and $h_{2} / M_{2}$, respectively, have positive constant sectional curvature. We have constructed a special I-parameter family of Riemannian metrics $d(t)$ on $M_{1} \times M_{2}$ which is the deformation of the product metric $h_{1} / M_{1} \times h_{2} / M_{2}$ and it has strictly positive sectional curvature. In other words, we have proved that $\forall P \in M_{1} \times M_{2}$ the derivative of the sectional curvature with respect to the parameter $t$ for $t=0$ and for any plane which is spanned by $X \in\left(M_{1}\right)_{p}$ and $Y \in\left(M_{2}\right)_{p}$ is strictly positive.

Let $S^{2}$ be a two-dimensional sphere with the canonical metric $g$ whose sectional curvature is positive constant. Consider the product of two manifolds $S^{2} \times S^{2}$. It is not known, ([1], p. 287), ([4], p, 171), ([11], p. 106), if there exists a deformation of the metric $g \times g$ with strictly positive sectional curvature.

Let $\boldsymbol{R}^{2}$ be a two-dimensional Euclidean space with the metric $h$ induced from the canonical metric $g$ of $S^{2}$. It is obvious that the Riemannian manifold $\boldsymbol{R}^{2}$ with the metric $h$ has constant sectional curvature. Consider two such Riemannian manifolds $\left(\boldsymbol{R}_{1}^{2}, h_{1}\right),\left(\boldsymbol{R}_{2}^{2}, h_{2}\right)$. The space $\boldsymbol{R}_{1}^{2} \times \boldsymbol{R}_{2}^{2}$ with the metric $h_{1} \times h_{2}$ has no negative sectional curvature. I do not know if there is a deformation of the metric $h_{1} \times h_{2}$ whose sectional curvature is strictly positive.

1. Let $\boldsymbol{R}^{2}$ be a Euclidean plane which is referred to a coordinate system $\left(u_{1}, u_{2}\right)$ on which we obtain a metric defined by

$$
h_{1}=\left\{h_{11}=1, h_{12}=h_{21}=0, h_{22}=\sin ^{2} u_{1}\right\},
$$

whose sectional curvature is positive constant 1 .

Consider an open Riemannian submanifold $M_{1}$ of the Riemannian manifold $\left(\boldsymbol{R}_{1}^{2}, h_{1}\right)$ defined by

$$
M_{1}=\left\{\left(u_{1}, u_{2}\right) \in \boldsymbol{R}_{1}^{2}: 0<u_{1}<\frac{\pi}{2},-\infty<u_{2}<\infty\right\},
$$

whose metric is $h_{1} / M_{1}$.

Let $\boldsymbol{R}_{2}^{2}$ be also another Euclidean plane referred to a coordinate system $\left(u_{3}, u_{4}\right)$ on which we take a metric defined by 


$$
h_{2}=\left\{h_{33}=1, h_{34}=h_{43}=0, h_{44}=\sin ^{2} u_{3}\right\} .
$$

We also consider an open Riemannian submanifold $M_{2}$ of $\boldsymbol{R}_{2}^{2}$ defined by

$$
M_{2}=\left\{\left(u_{3}, u_{4}\right) \in \boldsymbol{R}^{2}: 0<u_{3}<\frac{\pi}{2},-\infty<u_{4}<\infty\right\},
$$

whose metric is $h_{2} / M_{2}$.

Let $M_{1} \times M_{2}$ be the product manifold of $M_{1}, M_{2}$ which is defined by

$$
\begin{array}{r}
M_{1} \times M_{2}=\left\{\left(u_{1}, u_{2}, u_{3}, u_{4}\right) \in \boldsymbol{R}_{1}^{2} \times \boldsymbol{R}_{2}^{2}: 0<u_{1}<\frac{\pi}{2},\right. \\
\left.-\infty<u_{2}<\infty, 0<u_{3}<\frac{\pi}{2},-\infty<u_{4}<\infty\right\} .
\end{array}
$$

On the manifold $M_{1} \times M_{2}$ we get a special 1-parameter family of Riemannian metrics defined by

$$
d(t)=\left\{\begin{array}{l}
d_{11}=1+t f_{1}, d_{22}=\sin ^{2} u_{1}\left(1+t f_{2}\right), \\
d_{33}=1+t \varphi_{1}, d_{44}=\sin ^{2} u_{3}\left(1+t \varphi_{2}\right), d_{i j}=0, \text { if } i \neq j
\end{array}\right.
$$

where

$f_{1}=f_{1}\left(u_{3}, u_{4}\right), f_{2}=f_{2}\left(u_{3}, u_{4}\right), \varphi_{1}=\varphi_{1}\left(u_{1}, u_{2}\right), \varphi_{2}=\varphi_{2}\left(u_{1}, u_{2}\right),-\varepsilon<t<\varepsilon$, $\varepsilon$ is a small positive number.

It is obvious that $d(0)=h_{1} / \mathbf{M}_{1} \times h_{2} / M_{2}$.

2. Let $P$ be any point of $M_{1} \times M_{2}$. As is known, the sectional curvature of a plane spanned two vectors $X, Y$ of the tangent space $\left(M_{1} \times M_{2}\right)_{P}$ is given by

$$
\sigma(X, Y)(t)=-\frac{\langle R(X, Y) X, Y\rangle}{\|X\|^{2}\|Y\|^{2}-\langle X, Y\rangle^{2}}
$$

If we apply Taylor's expansion theorem for the function $\sigma(X, Y)(t)$, we get

$$
\sigma(X, Y)(t)=\sigma(X, Y)(0)+\sigma_{t}^{\prime}(X, Y)(0) \frac{t}{1}+\sigma_{t}^{\prime \prime}(X, Y)(0) \frac{t^{2}}{2 !}+\cdots .
$$

From the above formula we conclude that the sign of $\sigma(X, Y)(t)$ depends on the sign of $\sigma(X, Y)(0)$, if $t$ is a small positive number and $\sigma(X, Y)(0) \neq 0$, but if $\sigma(X, Y)=0$, then its sign depends on $t \sigma_{t}^{\prime}(X, Y)(0)$.

As is known ([1], p. 287), $\sigma(X, Y)(0)=0$, if $X \in\left(M_{1}\right)_{P}$ and $Y \in\left(M_{2}\right)_{P}$. In this case we estimate $\sigma(X, Y)(t)$ which is given by the formula

$$
\sigma(X, Y)(t)=-\frac{A(t)}{B(t)}
$$


where

$$
\begin{aligned}
A(t) & =\langle R(X, Y) X, Y\rangle=R_{1313}\left(X^{1}\right)^{2}\left(Y^{3}\right)^{2}+R_{1414}\left(X^{1}\right)^{2}\left(Y^{4}\right)^{2} \\
& +R_{2323}\left(X^{2}\right)^{2}\left(Y^{3}\right)^{2}+R_{2424}\left(X^{2}\right)^{2}\left(Y^{4}\right)^{2}+2 R_{1323} X^{1} X^{2}\left(Y^{3}\right)^{2} \\
& +2 R_{1314}\left(X^{1}\right)^{2} Y^{3} Y^{4}+2 R_{2324}\left(X^{2}\right)^{2} Y^{3} Y^{4}+2 R_{1424} X^{1} X^{2}\left(Y^{4}\right)^{2} \\
& +2\left(R_{1324}+R_{1423}\right) X^{1} X^{2} Y^{3} Y^{4} .
\end{aligned}
$$

because, in this case, $\langle X, Y\rangle=0$.

From relation (2.1), we obtain

$$
\sigma(X, Y)(0)=-\frac{A(0)}{B(0)}=0
$$

or

$$
A(0)=0 \text {. }
$$

If we differentiate the same relation (2.1) with respect to $t$, we obtain

$$
\sigma_{t}^{\prime}(X, Y)(0)=-\frac{A^{\prime}(0) B(0)-A(0) B^{\prime}(0)}{B^{2}(0)},
$$

which, by virtue of (2.4), takes the form

$$
\sigma_{t}^{\prime}(X, Y)(0)=-\frac{A^{\prime}(0)}{B(0)}
$$

From the formula (2.2), we obtain

$$
\begin{aligned}
A^{\prime}(0) & =R_{1313}^{\prime}(0)\left(X^{1}\right)^{2}\left(Y^{3}\right)^{2}+R_{2323}^{\prime}(0)\left(X^{2}\right)^{2}\left(Y^{3}\right)^{2}+R_{1414}^{\prime}(0)\left(X^{1}\right)^{2}\left(Y^{4}\right)^{2} \\
& +R_{2424}^{\prime}(0)\left(X^{2}\right)^{2}\left(Y^{4}\right)^{2}+2 R_{1323}^{\prime}(0) X^{1} X^{2}\left(Y^{3}\right)^{2}+2 R_{1314}^{\prime}(0)\left(X^{1}\right)^{2} Y^{3} Y^{4} \\
& +2 R_{2324}^{\prime}(0)\left(X^{2}\right)^{2} Y^{3} Y^{4}+2 R_{1424}^{\prime}(0) X^{1} X^{2}\left(Y^{4}\right)^{2} \\
& +2\left\{R_{1324}^{\prime}(0)+R_{1423}^{\prime}(0)\right\} X^{1} X^{2} Y^{3} Y^{4}
\end{aligned}
$$

We shall estimate the coefficients of the Riemannian tensor which appear in the formula (2.6). As is known, $R_{i j k l}$ is given by ([18], p. 18)

$$
\begin{aligned}
R_{i j k l}= & \frac{1}{2}\left\{\frac{\partial^{2} d_{i k}}{\partial u_{j} \partial u_{l}}+\frac{\partial^{2} d_{j l}}{\partial u_{i} \partial u_{k}}-\frac{\partial^{2} d_{j k}}{\partial u_{i} \partial u_{l}}-\frac{\partial^{2} d_{i l}}{\partial u_{j} \partial u_{k}}\right\} \\
& -d_{r s}\left\{\Gamma_{j k}^{r} \Gamma_{i l}^{s}-\Gamma_{j l}^{r} \Gamma_{i k}^{r}\right\},
\end{aligned}
$$

where $\Gamma_{j k}^{r}, \Gamma_{i l}^{s}, \Gamma_{j l}^{r}, \Gamma_{i k}^{s}$ are the Christoffel symbols of second kind. From (1.1) and (2.7), if we make the calculations, we obtain 


$$
\begin{aligned}
& R_{1313}=\frac{t}{2}\left(\frac{\partial^{2} f_{1}}{\partial u_{3}^{2}}+\frac{\partial^{2} \varphi_{1}}{\partial u_{1}^{2}}\right)-\frac{t^{2}}{4}\left\{\frac{\left(\partial f_{1} / \partial u_{3}\right)^{2}}{1+t f_{1}}+\frac{\left(\partial \varphi_{1} / \partial u_{1}\right)^{2}}{1+t \varphi_{1}}\right\}, \\
& R_{1414}=\frac{t}{2}\left(\frac{\partial^{2} f_{1}}{\partial u_{4}^{2}}+\sin ^{2} u_{3} \frac{\partial^{2} \varphi_{2}}{\partial u_{1}^{2}}+\frac{\sin 2 u_{3}\left(\partial f_{1} / \partial u_{3}\right)}{2\left(1+t \varphi_{1}\right)}\right) \\
& -\frac{t^{2}}{4}\left\{\frac{\left(\partial f_{1} / \partial u_{4}\right)^{2}}{1+t f_{1}}+\frac{\sin ^{2} u_{3}\left(\partial \varphi_{2} / \partial u_{1}\right)^{2}}{1+t f_{2}}-\frac{\sin 2 u_{3}\left(\partial f_{1} / \partial u_{3}\right) \varphi_{2}}{1+t \varphi_{1}}\right\}, \\
& R_{2323}=\frac{t}{2}\left(\sin ^{2} u_{1} \frac{\partial^{2} f_{2}}{\partial u_{3}^{2}}+\frac{\partial^{2} \varphi_{1}}{\partial u_{2}^{2}}+\frac{\sin 2 u_{1}\left(\partial \varphi_{1} / \partial u_{1}\right)}{2\left(1+t f_{1}\right)}\right) \\
& -\frac{t^{2}}{4}\left\{\frac{\sin ^{2} u_{1}\left(\partial f_{2} / \partial u_{3}\right)^{2}}{1+t f_{2}}+\frac{\left(\partial \varphi_{1} / \partial u_{2}\right)^{2}}{1+t \varphi_{1}}-\frac{\sin 2 u_{1} f_{2}\left(\partial \varphi_{1} / \partial u_{1}\right)}{1+t f_{1}}\right\}, \\
& R_{2424}=\frac{t}{2}\left(\sin ^{2} u_{1} \frac{\partial^{2} f_{2}}{\partial u_{4}^{2}}+\sin ^{2} u_{3} \frac{\partial^{2} \phi_{2}}{\partial u_{2}^{2}}+\frac{\sin 2 u_{1} \sin ^{2} u_{3}\left(\partial \varphi_{2} / \partial u_{1}\right)}{2\left(1+t f_{1}\right)}\right. \\
& +\frac{\sin 2 u_{3} \sin ^{2} u_{1}\left(\partial f_{2} / \partial u_{3}\right)}{2\left(1+t \varphi_{1}\right)}-\frac{t^{2}}{4}\left\{\frac{\sin ^{2} u_{1}\left(\partial f_{2} / \partial u_{4}\right)^{2}}{1+t f_{2}}\right. \\
& +\frac{\sin ^{2} u_{3}\left(\partial \varphi_{2} / \partial u_{3}\right)^{2}}{1+t \varphi_{2}}-\frac{\sin 2 u_{1} \sin ^{2} u_{3} f_{2}\left(\partial \varphi_{2} / \partial u_{1}\right)}{1+t f_{1}} \\
& \left.-\frac{\sin 2 u_{3} \sin ^{2} u_{1} \varphi_{2}\left(\partial f_{2} / \partial u_{:}\right)}{1+t \varphi_{1}}\right\} \text {, } \\
& R_{1323}=\frac{t}{2}\left(\frac{\partial^{2} \varphi_{1}}{\partial u_{1} \partial u_{2}}-2 \frac{\cos u_{1}}{\sin u_{1}} \frac{\partial \varphi_{1}}{\partial u_{2}}\right)-\frac{t^{2}}{4} \frac{\left(\partial \varphi_{1} / \partial u_{1}\right)\left(\partial \varphi_{1} / \partial u_{2}\right)}{1+t \varphi_{1}}, \\
& R_{1314}=\frac{t}{2}\left(\frac{\partial^{2} f_{1}}{\partial u_{3} \partial u_{4}}-2 \frac{\cos u_{3}}{\sin u_{3}} \frac{\partial f_{1}}{\partial u_{4}}\right)-\frac{t^{2}}{4} \frac{\left(\partial f_{1} / \partial u_{3}\right)\left(\partial f_{1} / \partial u_{4}\right)}{1+t f_{1}} \text {, } \\
& R_{2324}=\frac{t}{2} \sin ^{2} u_{1}\left(\frac{\partial^{2} f_{2}}{\partial u_{3} \partial u_{4}}-\frac{\cos u_{3}}{\sin u_{3}} \frac{\partial f_{2}}{\partial u_{4}}\right) \\
& -\frac{t^{2}}{4} \frac{\sin ^{2} u_{1}\left(\partial f_{2} / \partial u_{3}\right)\left(\partial f_{2} / \partial u_{4}\right)}{1+t f_{2}} \\
& R_{1424}=\frac{t}{2} \sin ^{2} u_{3}\left(\frac{\partial^{2} \varphi_{2}}{\partial u_{1} \partial u_{2}}-\frac{\cos u_{1}}{\sin u_{1}} \frac{\partial \varphi_{2}}{\partial u_{2}}\right) \\
& -\frac{t^{2}}{4} \frac{\sin ^{2} u_{3}\left(\partial \varphi_{2} / \partial u_{1}\right)\left(\partial \varphi_{2} / \partial u_{2}\right)}{1+t \varphi_{2}} \\
& R_{1324}=R_{1423}=0 \text {. }
\end{aligned}
$$

If we choose the functions $\varphi_{1}, f_{1}, f_{2}, \varphi_{2}$ such that they satisfy the partial differential equations 


$$
\begin{aligned}
& \frac{\partial^{2} \varphi_{1}}{\partial u_{1} \partial u_{2}}-2 \frac{\cos u_{1}}{\sin u_{1}} \frac{\partial \varphi_{1}}{\partial u_{2}}=0, \\
& \frac{\partial^{2} f_{1}}{\partial u_{3} \partial u_{4}}-2 \frac{\cos u_{3}}{\sin u_{3}} \frac{\partial f_{1}}{\partial u_{4}}=0, \\
& \frac{\partial^{2} f_{2}}{\partial u_{3} \partial u_{4}}-\frac{\cos u_{3}}{\sin u_{3}} \frac{\partial f_{2}}{\partial u_{4}}=0, \\
& \frac{\partial^{2} \varphi_{2}}{\partial u_{1} \partial u_{2}}-\frac{\cos u_{1}}{\sin u_{1}} \frac{\partial \varphi_{2}}{\partial u_{2}}=0,
\end{aligned}
$$

then the formulas (2.9) take the form

$$
\begin{aligned}
& R_{1323}=-\frac{t^{2}}{4} \frac{\left(\partial \varphi_{1} / \partial u_{1}\right)\left(\partial \varphi_{1} / \partial u_{2}\right)}{1+t \varphi_{1}}, \\
& R_{1314}=-\frac{t^{2}}{4} \frac{\left(\partial f_{1} / \partial u_{3}\right)\left(\partial f_{1} / \partial u_{4}\right)}{1+t f_{1}}, \\
& R_{2324}=-\frac{t^{2}}{4} \frac{\sin ^{2} u_{1}\left(\partial f_{2} / \partial u_{3}\right)\left(\partial f_{2} / \partial u_{4}\right)}{1+t f_{2}}, \\
& R_{1424}=-\frac{t^{2}}{4} \frac{\sin ^{2} u_{3}\left(\partial \varphi_{2} / \partial u_{1}\right)\left(\partial \varphi_{2} / \partial u_{2}\right)}{1+t \varphi_{2}} .
\end{aligned}
$$

From the relations (2.8) and (2.12) we obtain

$$
\begin{aligned}
& R_{3313}^{\prime}(0)= \frac{1}{2}\left(\frac{\partial^{2} f_{1}}{\partial u_{3}^{2}}+\frac{\partial^{2} \varphi_{1}}{\partial u_{1}{ }^{2}}\right), \\
& R_{1414}^{\prime}(0)= \frac{1}{2}\left(\frac{\partial^{2} f_{1}}{\partial u_{4}{ }^{2}}+\sin ^{2} u_{3} \frac{\partial^{2} \varphi_{2}}{\partial u_{1}{ }^{2}}+\frac{\sin 2 u_{3}}{2} \frac{\partial f_{1}}{\partial u_{3}}\right), \\
& R_{2323}^{\prime}(0)= \frac{1}{2}\left(\frac{\partial^{2} \varphi_{1}}{\partial u_{1}^{2}}+\sin ^{2} u_{1} \frac{\partial^{2} f_{2}}{\partial u_{3}^{2}}+\frac{\sin 2 u_{1}}{2} \frac{\partial \varphi_{1}}{\partial u_{1}}\right), \\
& R_{2444}^{\prime}(0)= \frac{1}{2}\left(\sin ^{2} u_{1} \frac{\partial^{2} f_{2}}{\partial u_{4}^{2}}+\sin ^{2} u_{3} \frac{\partial^{2} \varphi_{2}}{\partial u_{2}{ }^{2}}+\frac{\sin 2 u_{1} \sin ^{2} u_{3}}{2} \frac{\partial \varphi_{2}}{\partial u_{1}}\right. \\
&\left.+\frac{\sin 2 u_{3} \sin ^{2} u_{1}}{2} \frac{\partial f_{2}}{\partial u_{3}}\right) \cdot \\
& R_{1323}^{\prime}(0)=R_{1314}^{\prime}(0)=R_{2324}^{\prime}(0)=R_{1424}^{\prime}(0)=0 .
\end{aligned}
$$

The first partial differential equation of (2.11) can be written

$$
\frac{\partial^{2} \varphi_{1}}{\partial u_{1} \partial u_{2}}-\frac{\partial}{\partial u_{1}} \log \sin ^{2} u_{1} \frac{\partial \varphi_{1}}{\partial u_{2}}=0,
$$

or

$$
\frac{\partial^{2} \varphi_{1} / \partial u_{1} \partial u_{2}}{\partial \varphi_{1} / \partial u_{2}}=\frac{\partial}{\partial u_{1}} \log \sin ^{2} u_{1},
$$

or

$$
\frac{\partial \varphi_{1}}{\partial u_{2}}=Z\left(u_{2}\right) \sin ^{2} u_{1},
$$


whose general solution is

$$
\varphi_{1}=V_{1}\left(u_{2}\right) \sin ^{2} u_{1}+T_{1}\left(u_{1}\right),
$$

where $V_{1}\left(u_{2}\right)$ and $T_{1}\left(u_{1}\right)$ are arbitrary functions of $u_{2}$ and $u_{1}$, respectively.

We can find the general solutions of the rest of partial differential equations (2.11) in the same way. The general solutions of these equations are

$$
\begin{aligned}
& f_{1}=\sin ^{2} u_{3} \lambda_{1}\left(u_{4}\right)+\mu_{1}\left(u_{3}\right), \\
& \varphi_{2}=\sin u_{1} V_{2}\left(u_{2}\right)+T_{2}\left(u_{1}\right), \\
& f_{2}=\sin u_{3} \lambda_{2}\left(u_{4}\right)+\mu_{2}\left(u_{3}\right),
\end{aligned}
$$

where $\lambda_{1}\left(u_{4}\right), \mu_{1}\left(u_{3}\right), V_{2}\left(u_{2}\right), T_{2}\left(u_{1}\right), \lambda_{2}\left(u_{4}\right), \mu\left(u_{3}\right)$ are arbitrary functions of $u_{4}, u_{3}, u_{2}, u_{1}, u_{4}, u_{3}$, respectively.

The formulas (2.13) by virtue of (2.15) and (2.16) take the form

$$
\begin{aligned}
R_{1313}^{\prime}(0)= & \frac{1}{2}\left\{2 \cos 2 u_{1} V_{1}\left(u_{2}\right)+T_{1}^{\prime \prime}\left(u_{1}\right)\right\}+\frac{1}{2}\left\{2 \cos 2 u_{3} \lambda_{1}\left(u_{4}\right)\right. \\
& \left.+\mu_{1}^{\prime \prime}\left(u_{3}\right)\right\}, \\
R_{1414}^{\prime}(0)= & \frac{1}{2}\left\{\sin ^{2} u_{3}\left(\lambda_{1}^{\prime \prime}\left(u_{4}\right)+T_{2}^{\prime \prime}\left(u_{1}\right)\right)+\frac{\sin ^{2} 2 u_{3}}{2} \lambda_{1}\left(u_{4}\right)\right. \\
& \left.+\frac{\sin 2 u_{3}}{2} \mu_{1}^{\prime}\left(u_{3}\right)-\sin ^{2} u_{3} \sin u_{1} V_{2}\left(u_{2}\right)\right\},
\end{aligned}
$$

$$
\begin{aligned}
R_{2323}^{\prime}(0)= & \frac{1}{2}\left\{\sin ^{2} u_{1}\left(\mu_{2}^{\prime \prime}\left(u_{3}\right)+V_{1}^{\prime \prime}\left(u_{2}\right)\right)+\frac{\sin ^{2} 2 u_{1}}{2} V_{1}\left(u_{2}\right)\right. \\
& \left.+\frac{\sin 2 u_{1}}{2} T_{1}^{\prime}\left(u_{1}\right)-\sin ^{2} u_{1} \sin u_{3} \lambda_{2}\left(u_{4}\right)\right\}, \\
R_{2424}^{\prime}(0)= & \frac{\sin ^{2} u_{1} \sin u_{3}}{2}\left\{\lambda_{2}^{\prime \prime}\left(u_{4}\right)+\cos u_{3} \mu_{2}^{\prime}\left(u_{3}\right)+\cos ^{2} u_{3} \lambda_{2}\left(u_{4}\right)\right. \\
& +\frac{\sin u_{1} \sin ^{2} u_{3}}{2}\left\{V_{2}^{\prime \prime}\left(u_{2}\right)+\cos u_{1} T_{2}^{\prime}\left(u_{1}\right)+\cos ^{2} u_{1} V_{2}\left(u_{2}\right)\right\} .
\end{aligned}
$$

The relation (2.6) by means of (2.10) and (2.14) takes the form

$$
\begin{aligned}
A^{\prime}(0)= & R_{1313}^{\prime}(0)\left(X^{1}\right)^{2}\left(Y^{3}\right)^{2}+R_{2323}^{\prime}(0)\left(X^{2}\right)^{2}\left(Y^{3}\right)^{2}+R_{1414}^{\prime}(0)\left(X^{1}\right)^{2}\left(Y^{4}\right)^{2} \\
& +R_{2424}^{\prime}(0)\left(X^{2}\right)^{2}\left(Y^{4}\right)^{2}
\end{aligned}
$$

In order that $\sigma^{\prime}(X, Y)(0)=-A^{\prime}(0) / B(0)$ be positive on the Riemannian manifold $M_{1} \times M_{2}$, it must be

$$
\mathrm{A}^{\prime}(0)<0 \text {. }
$$

From the formula (2.18) we conclude that (2.19) is valid when we have 


$$
R_{1313}^{\prime}(0)<0, \quad R_{1414}^{\prime}(0)<0, \quad R_{2323}^{\prime}(0)<0, \quad R_{2424}^{\prime}(0)<0,
$$

which, by virtue of (2.17), take the form

$$
\begin{aligned}
& \frac{1}{2}\left\{2 \cos 2 u_{1} V_{1}\left(u_{2}\right)+T_{1}^{\prime \prime}\left(u_{1}\right)\right\}+\frac{1}{2}\left\{2 \cos 2 u_{3} \lambda_{1}\left(u_{4}\right)+\mu_{1}^{\prime \prime}\left(u_{3}\right)\right\}<0, \\
& \frac{1}{2}\left\{\sin ^{2} u_{3}\left(\lambda_{1}^{\prime \prime}\left(u_{4}\right)+T_{2}^{\prime \prime}\left(u_{1}\right)\right)+\frac{\sin ^{2} 2 u_{3}}{2} \lambda_{1}\left(u_{4}\right)+\frac{\sin 2 u_{3}}{2} \mu_{1}^{\prime}\left(u_{3}\right)\right. \\
& \left.-\sin ^{2} u_{3} \sin u_{1} V_{2}\left(u_{2}\right)\right\}<0, \\
& \frac{1}{2}\left\{\sin ^{2} u_{1}\left(\mu_{2}^{\prime \prime}\left(u_{3}\right)+V_{1}^{\prime \prime}\left(u_{2}\right)\right)+\frac{\sin ^{2} 2 u_{1}}{2} V_{1}\left(u_{2}\right)+\frac{\sin 2 u_{1}}{2} T_{1}^{\prime}\left(u_{1}\right)\right. \\
& \left.-\sin ^{2} u_{1} \sin u_{3} \lambda_{2}\left(u_{4}\right)\right\}<0, \\
& \frac{\sin ^{2} u_{1} \sin u_{3}}{2}\left\{\lambda_{2}^{\prime \prime}\left(u_{4}\right)+\cos u_{3} \mu_{2}^{\prime}\left(u_{3}\right)+\cos ^{2} u_{3} \lambda_{2}\left(u_{4}\right)\right\} \\
& \left.\left.+\frac{\sin u_{1} \sin ^{2} u_{3}}{2}\right\} V_{2}^{\prime \prime}\left(u_{2}\right)+\cos u_{1} T_{2}^{\prime}\left(u_{1}\right)+\cos ^{2} u_{1} V_{2}\left(u_{2}\right)\right\}<0,
\end{aligned}
$$

which must be valid on the Riemannian manifold $M_{1} \times M_{2}$. The above inequalities hold if we have

$$
\begin{aligned}
& 2 \cos 2 u_{1} V_{1}\left(u_{2}\right)+T_{1}^{\prime \prime}\left(u_{1}\right)<0, \\
& \sin ^{2} u_{1}\left(\mu_{2}^{\prime \prime}\left(u_{3}\right)+V_{1}^{\prime \prime}\left(u_{2}\right)\right)+\frac{\sin ^{2} 2 u_{1}}{2} V_{1}\left(u_{2}\right)+\frac{\sin 2 u_{1}}{2} T_{1}^{\prime}\left(u_{1}\right) \\
& \quad-\sin ^{2} u_{1} \sin u_{3} \lambda_{2}\left(u_{4}\right)<0, \\
& \lambda_{2}^{\prime \prime}\left(u_{4}\right)+\cos u_{3} \mu_{2}^{\prime}\left(u_{3}\right)+\cos ^{2} u_{3} \lambda_{2}\left(u_{4}\right)<0, \\
& 2 \cos 2 u_{3} \lambda_{1}\left(u_{4}\right)+\mu_{1}^{\prime \prime}\left(u_{3}\right)<0, \\
& \sin ^{2} u_{3}\left(\lambda_{1}^{\prime \prime}\left(u_{4}\right)+T_{2}^{\prime \prime}\left(u_{1}\right)\right)+\frac{\sin ^{2} 2 u_{3}}{2} \lambda_{1}\left(u_{4}\right)+\frac{\sin 2 u_{3}}{2} \mu_{1}^{\prime}\left(u_{3}\right) \\
& \quad-\sin ^{2} u_{3} \sin u_{1} V_{2}\left(u_{2}\right)<0, \\
& V_{2}^{\prime \prime}\left(u_{2}\right)+\cos u_{1} T_{2}^{\prime}\left(u_{1}\right)+\cos ^{2} u_{1} V_{2}\left(u_{2}\right)<0 .
\end{aligned}
$$

The inequalities (2.21) are similar to the inequalities (2.20); for this reason we shall only study the inequalities (2.20).

The factor $\cos 2 u_{1}$ changes sign when $0<u_{1}<2 / \pi$; from this and from the fact that $V_{1}\left(u_{2}\right)$ and $V_{1}^{\prime \prime}\left(u_{2}\right)$ must have constant sign and bounded when $-\infty<u_{2}<\infty$, we conclude that $V_{1}\left(u_{2}\right)$ must be a constant negative number $-\alpha$.

From the above remark, the inequalities (2.20) take the form 


$$
-2 \alpha \cos 2 u_{1}+T_{1}^{\prime \prime}\left(u_{1}\right)<0,
$$

(2.22) $\sin ^{2} u_{1} \mu_{2}^{\prime \prime}\left(u_{3}\right)-\alpha \frac{\sin ^{2} 2 u_{1}}{2}+\frac{\sin 2 u_{1}}{2} T_{1}^{\prime}\left(u_{1}\right)-\sin ^{2} u_{1} \sin u_{3} \lambda_{2}\left(u_{4}\right)<0$,

$$
\lambda_{2}^{\prime \prime}\left(u_{4}\right)+\cos u_{3} \mu_{2}^{\prime}\left(u_{3}\right)+\cos ^{2} u_{3} \lambda_{2}\left(u_{4}\right)<0 .
$$

In order for the second and the third inequalities of (2.22) to be valid, the function $\lambda_{2}\left(u_{4}\right)$ must be a positive constant number $\beta$.

Therefore the above inequalities become

$$
-2 \alpha \cos 2 u_{1}+T_{1}^{\prime \prime}\left(u_{1}\right)<0,
$$

(2.23) $\sin ^{2} u_{1} \ell_{2}^{\prime \prime}\left(u_{3}\right)-\frac{\alpha \sin ^{2} 2 u_{1}}{2}+\frac{\sin 2 u_{1}}{2} T_{1}^{\prime}\left(u_{1}\right)-\beta \sin ^{2} u_{1} \sin u_{3}<0$,

$$
\mu_{2}^{\prime}\left(u_{3}\right)+\beta \cos u_{3}<0 .
$$

If the functions $T_{1}\left(u_{1}\right), \mu_{2}\left(u_{3}\right)$ are chosen such that

$$
\begin{array}{ll}
T_{1}^{\prime}\left(u_{1}\right)<0, \quad \max \left\{T_{1}^{\prime \prime}\left(u_{1}\right)\right\}<-2 \alpha, & 0<u_{1}<\frac{\pi}{2}, \\
\max \left\{\mu_{2}^{\prime}\left(u_{3}\right)\right\}<-\beta, \quad \mu_{2}^{\prime \prime}\left(u_{3}\right)<0, & 0<u_{3}<\frac{\pi}{2},
\end{array}
$$

then the inequalities (2.23) hold.

We also conclude that if the functions $\lambda_{1}\left(u_{4}\right), V_{2}\left(u_{2}\right), \mu_{1}\left(u_{3}\right), T_{2}\left(u_{1}\right)$ satisfy the conditions

$$
\begin{gathered}
\lambda_{1}\left(u_{4}\right)=-\gamma, \quad V_{2}\left(u_{2}\right)=\delta, \\
\mu_{1}^{\prime}\left(u_{3}\right)<0, \quad \max \left\{\mu_{1}^{\prime \prime}\left(u_{3}\right)\right\}<-2 \gamma, \quad 0<u_{3}<\frac{\pi}{2}, \\
\max \left\{T_{2}^{\prime}\left(u_{1}\right)\right\}<-\delta, \quad T_{2}^{\prime \prime}\left(u_{1}\right)<0, \quad 0<u_{1}<\frac{\pi}{2},
\end{gathered}
$$

then the inequalities (2.21) hold.

Therefore, if the functions $\varphi_{1}, f_{1}, \varphi_{2}, f_{2}$ have the form

$$
\begin{array}{ll}
\varphi_{1}=-\alpha \sin ^{2} u_{1}+T_{1}\left(u_{1}\right), & \alpha>0, \\
f_{1}=-\gamma \sin ^{2} u_{3}+\mu_{1}\left(u_{3}\right), & \gamma>0, \\
\varphi_{2}=\delta \sin u_{1}+T_{2}\left(u_{1}\right), & \delta>0, \\
f_{2}=\beta \sin u_{3}+\mu_{2}\left(u_{3}\right), & \beta>0,
\end{array}
$$

such that the functions $T_{1}\left(u_{1}\right), \mu_{1}\left(u_{3}\right), T_{2}\left(u_{1}\right)$ and $\mu_{2}\left(u_{3}\right)$ satisfy the conditions 


$$
\begin{array}{ll}
T_{1}^{\prime}\left(u_{1}\right)<0, \quad \max \left\{T_{1}^{\prime \prime}\left(u_{1}\right)\right\}<-2 \alpha, & 0<u_{1}<\frac{\pi}{2}, \\
\max \left\{\mu_{2}^{\prime}\left(u_{3}\right)\right\}<-\beta, \quad \mu_{2}^{\prime \prime}\left(u_{3}\right)<0, & 0<u_{3}<\frac{\pi}{2}, \\
\mu_{1}^{\prime}\left(u_{3}\right)<0, \quad \max \left\{\mu_{1}^{\prime \prime}\left(u_{3}\right)\right\}<-2 \gamma, & 0<u_{3}<\frac{\pi}{2}, \\
\max \left\{T_{2}^{\prime}\left(u_{1}\right)\right\}<-\delta, \quad T_{2}^{\prime \prime}\left(u_{1}\right)<0, & 0<u_{1}<\frac{\pi}{2},
\end{array}
$$

then $\sigma_{t}^{\prime}(X, Y)(0)>0$ for $X \in\left(M_{1}\right)_{P}, Y \in\left(M_{2}\right)_{P}$.

Hence we have the following theorem.

Theorem. Let $M_{1}, M_{2}$ be two Riemannian spaces with positive constant sectional curvature defined in $\$ 1$. If we consider a special 1-parameter family of Riemannian metrics $d(t)$ on $M_{1} \times M_{2}$ defined by (1.1) where the functions $f_{1}, f_{2}, \varphi_{1}, \varphi_{2}$ have the form (2.24) in which the functions $T_{1}\left(u_{1}\right), \mu_{1}\left(u_{3}\right), T_{2}\left(u_{1}\right)$ and $\mu_{2}\left(u_{3}\right)$ must satisfy the conditions (2.25), then $\forall P \in M_{1} \times M_{2}$ the derivative of the sectional curvature of any plane spanned by $X \in\left(M_{1}\right)_{P}$ and $Y \in\left(M_{2}\right)_{P}$ with respect to $t$ for $t=0$ is strictly positive.

From the above, we conclude that if the parameter $t$ is positive and small enough, then the corresponding Riemannian metric $d(t)$ defined by (1.1) on $M_{1} \times M_{2}$, where the functions $f_{1}, f_{2}, \varphi_{1}, \varphi_{2}$ have the form (2.24) in which the functions $T_{1}\left(u_{1}\right), \mu_{1}\left(u_{3}\right), T_{2}\left(u_{1}\right)$ and $\mu_{2}\left(u_{3}\right)$ must satisfy the conditions (2.25), has strictly positive sectional curvature.

3. We can extend the manifold $M_{1} \times M_{2}$ to a manifold

$$
N_{1} \times N_{2} \supset M_{1} \times M_{2}
$$

such that there is a deformation of another product metric on $N_{1} \times N_{2}$ which has strictly positive sectional curvature.

This method can be stated as follows. On the Euclidean plane $\boldsymbol{R}_{2}^{2}$ we obtain a metric which is given by

$$
\omega_{1}=\left\{\omega_{11}=1, \quad \omega_{12}=\omega_{21}=0, \quad \omega_{22}=\sin ^{2} \frac{u_{1}}{n}\right\},
$$

where $n$ is an integer $>1$. The sectional curvature of this metric is $1 / n^{2}$.

Now, consider an open Riemannian submanifold $N_{1}$ of the Riemannian manifold $\left(\boldsymbol{R}_{1}^{2}, \omega_{1}\right)$ defined by

$$
N_{1}=\left\{\left(u_{1}, u_{2}\right) \in \boldsymbol{R}_{1}^{2}: 0<u_{1}<n \frac{\pi}{2},-\infty<u_{2}<\infty\right\},
$$


whose metric is $\omega_{1} / N_{1}$.

Similarly, on the Euclidean plane $\boldsymbol{R}_{2}^{2}$, we obtain a metric which is given by

$$
\omega_{2}=\left\{\omega_{33}=1, \quad \omega_{34}=\omega_{43}=0, \omega_{44}=\sin ^{2} \frac{u_{3}}{n}\right\},
$$

whose sectional curvature is $1 / n^{2}$.

Let $N_{2}$ be an open Riemannian submanifold of the Riemannian manifold $\left(\boldsymbol{R}_{2}^{2}, \omega_{2}\right)$ which is defined by

$$
N_{2}=\left\{\left(u_{3}, u_{4}\right) \in \boldsymbol{R}_{2}^{2}: 0<u_{3}<n \frac{\pi}{2}, \quad-\infty<u_{4}<\infty\right\},
$$

whose metric is $\omega_{2} / N_{2}$.

We consider the product manifold $N_{1} \times N_{2}$ of $N_{1}, N_{2}$ defined by

$$
\begin{aligned}
& N_{1} \times N_{2}=\left\{\left(u_{1}, u_{2}, u_{3}, u_{4}\right) \in \boldsymbol{R}_{1}^{2} \times \boldsymbol{R}_{2}^{2}: 0<u_{1}<n \frac{\pi}{2}, \quad-\infty<u_{2}<\infty,\right. \\
& \left.0<u_{3}<n \frac{\pi}{2}, \quad-\infty<u_{4}<\infty\right\} .
\end{aligned}
$$

It is obvious that $\left(N_{1} \times N_{2}\right) \supset\left(M_{1} \times M_{2}\right)$ and with the same technique as in $\S 2$ we can prove that there is a deformation of the metric $\omega_{1} / N_{1} \times \omega_{2} / N_{2}$ which has strictly positive sectional curvature on the manifold $N_{1} \times N_{2}$.

Acknowledgment is due to Professor S. Kobayashi for many helpful suggestions.

\section{REFERENCES}

1. M. Berger, Variétés riemanniennes à courbure positite, Colloqué intern. C. N. R. S. Bul. Soc. Math., France 87 (1959), 285-292.

2. , Trois remarques sur variétés riemannienes à courbure positive C. R. Acad. Sci. Paris 263 (1966), 76-78.

3. B. Bishop and R. Crittenden, Geometry of manifolds, Academic Press, New York, 1964.

4. S. Chern, The geometry of G-structures, Bull. Amer. Math. Soc. 72 (1966), 167-219.

5. L. Eisenhart, Riemannian geometry, Princeton University Press, 1949.

6. T. Frankel, Manifolds with positive curvature, Pacific J. Math. 11 (1961), 165-174.

7. H. Guggenheimer, Differential geometry, McGraw-Hill Book Company, 1963.

8. S. Helgason, Differential geometry and symmetric spaces, Academic Press, New York, 1962.

9. N. Hicks, Notes on differential geometry, Math. Studies, No. 3, Van Nostrand, New York, 1965.

10. S. Kobayashi and K. Nomizu, Foundations of differential geometry, Vol. 1, Interscience, New York, 1963.

11. J. Milnor, Morse theory, Annals of Math. Studies, No. 51, Princeton University Press, 1963. 
12. S. Myers, Riemannian manifolds with positive mean curvature, Duke Math. J. 8 (1941), 401-404.

13. S. Sternberg, Lectures on differential geometry, Prentice-Hall, Englewood Cliffs, N. J., 1964.

14. G. Tsagas, A Riemannian space with strictly positive sectional curvature, Pacific J. Math. 25

15. Y. Tsukamoto, On Riemannian manifolds with positive curvature, Mem. Fac. Sci, Kyushu University 15 (1961), 90-96.

16. J. Wolf, Spaces of constant curvature, McGraw-Hill series in higher mathematics, 1967.

17. K. Yano, Differential geometry on complex and almost complex spaces, Pergamon Press, New York, 1965.

18. K. Yano and S. Bochner, Curvature and Betti numbers, Annals of Math. Studies, No. 32, Princeton University Press, 1953.

Received July 16, 1968.

UNIVERSITy OF CALIFORNIA, BERKELEY 



\section{PACIFIC JOURNAL OF MATHEMATICS}

\section{EDITORS}

H. ROYDEN

Stanford University

Stanford, California

\author{
R. R. Phelps \\ University of Washington \\ Seattle, Washington 98105
}

J. DugundJI

Department of Mathematics

University of Southern California

Los Angeles, California 90007

RICHARD ARENS

University of California

Los Angeles, California 90024

\section{ASSOCIATE EDITORS}

E. F. BECKenbaCH

B. H. NEUMANN

F. WOLF

K. YoSHIDA

\section{SUPPORTING INSTITUTIONS}

\author{
UNIVERSITY OF BRITISH COLUMBIA \\ CALIFORNIA INSTITUTE OF TECHNOLOGY \\ UNIVERSITY OF CALIFORNIA \\ MONTANA STATE UNIVERSITY \\ UNIVERSITY OF NEVADA \\ NEW MEXICO STATE UNIVERSITY \\ OREGON STATE UNIVERSITY \\ UNIVERSITY OF OREGON \\ OSAKA UNIVERSITY \\ UNIVERSITY OF SOUTHERN CALIFORNIA
}

\author{
STANFORD UNIVERSITY \\ UNIVERSITY OF TOKYO \\ UNIVERSITY OF UTAH \\ WASHINGTON STATE UNIVERSITY \\ UNIVERSITY OF WASHINGTON \\ $\stackrel{*}{*} \stackrel{*}{*} \stackrel{*}{*}$ AMERICAN MATHEMATICAL SOCIETY \\ CHEVRON RESEARCH CORPORATION \\ TRW SYSTEMS \\ NAVAL WEAPONS CENTER
}

The Supporting Institutions listed above contribute to the cost of publication of this Journal, but they are not owners or publishers and have no responsibility for its content or policies.

Mathematical papers intended for publication in the Pacific Journal of Mathematics should be in typed form or offset-reproduced, double spaced with large margins. Underline Greek letters in red, German in green, and script in blue. The first paragraph or two must be capable of being used separately as a synopsis of the entire paper. It should not contain references to the bibliography. Manuscripts, in duplicate if possible, may be sent to any one of the four editors. Please classify according to the scheme of Math. Rev. 36, 1539-1546. All other communications to the editors should be addressed to the managing editor, Richard Arens, University of California, Los Angeles, California, 90024.

50 reprints are provided free for each article; additional copies may be obtained at cost in multiples of 50 .

The Pacific Journal of Mathematics is published monthly. Effective with Volume 16 the price per volume (3 numbers) is $\$ 8.00$; single issues, $\$ 3.00$. Special price for current issues to individual faculty members of supporting institutions and to individual members of the American Mathematical Society: $\$ 4.00$ per volume; single issues $\$ 1.50$. Back numbers are available.

Subscriptions, orders for back numbers, and changes of address should be sent to Pacific Journal of Mathematics, 103 Highland Boulevard, Berkeley, California, 94708.

PUBLISHED BY PACIFIC JOURNAL OF MATHEMATICS, A NON-PROFIT CORPORATION

Printed at Kokusai Bunken Insatsusha (International Academic Printing Co., Ltd.), 7-17, Fujimi 2-chome, Chiyoda-ku, Tokyo, Japan. 


\section{Pacific Journal of Mathematics \\ Vol. 29, No. $3 \quad$ July, 1969}

Herbert James Alexander, Extending bounded holomorphic functions from certain subvarieties of a polydisc ...................... 485

Edward T. Cline, On an embedding property of generalized Carter

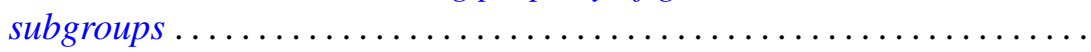

Roger Cuppens, On the decomposition of infinitely divisible characteristic functions with continuous Poisson spectrum. II ...............

William Richard Emerson, Translation kernels on discrete Abelian

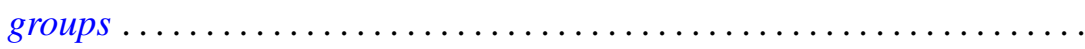

Robert William Gilmer, Jr., Power series rings over a Krull domain ....... 543

Julien O. Hennefeld, The Arens products and an imbedding theorem ...... 551

James Secord Howland, Embedded eigenvalues and virtual poles ........ 565

Bruce Ansgar Jensen, Infinite semigroups whose non-trivial homomorphs are all isomorphic .............................. 583

Michael Joseph Kascic, Jr., Polynomials in linear relations. II .......... 593

J. Gopala Krishna, Maximum term of a power series in one and several

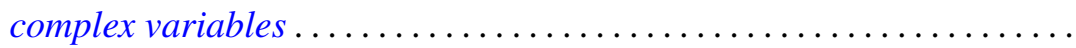

Renu Chakravarti Laskar, Eigenvalues of the adjacency matrix of cubic lattice graphs ...................................

Thomas Anthony Mc Cullough, Rational approximation on certain plane

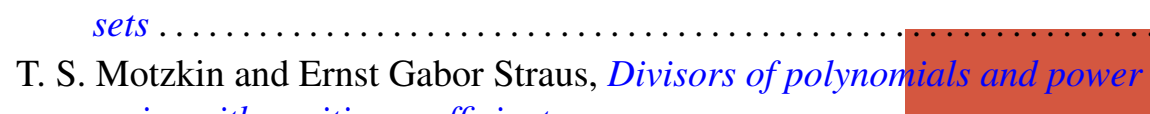
series with positive coefficients .

Graciano de Oliveira, Matrices with prescribed characteristic polynomial and a prescribed submatrix.

Graciano de Oliveira, Matrices with prescribed characteristic polynomial and a prescribed submatrix. II .

Donald Steven Passman, Exceptional 3/2-transitive permutation groups .................................

Grigorios Tsagas, A special deformation of the metric with no negative sectional curvature of a Riemannian space............

Joseph Zaks, Trivially extending decompositions of $E^{n}$ 